\title{
Nonequilibrium Cooper pairing in the nonadiabatic Regime
}

\author{
Emil A. Yuzbashyan ${ }^{1}$, Boris L. Altshuler ${ }^{2}$, Vadim B. Kuznetsov ${ }^{3}$, and Victor Z. Enolskii ${ }^{4}$ \\ ${ }^{1}$ Center for Materials Theory, Rutgers University, Piscataway, New Jersey 08854, USA \\ ${ }^{2}$ Physics Department, Princeton University, Princeton, NJ 08544, USA \\ ${ }^{3}$ Department of Applied Mathematics, University of Leeds, Leeds, LS2 9JT, UK \\ ${ }^{4}$ Department of Mathematics, Heriot-Watt University, Edinburgh EH14 4AS, UK
}

\begin{abstract}
We obtain a complete solution for the mean-field dynamics of the BCS paired state with a large, but finite number of Cooper pairs in the nonadiabatic regime. We show that the problem reduces to a classical integrable Hamiltonian system and derive a complete set of its integrals of motion. The condensate exhibits irregular multi-frequency oscillations ergodically exploring the part of the phase-space allowed by the conservation laws. In the thermodynamic limit however the system can asymptotically reach a steady state.
\end{abstract}

The study of the dynamics of the BCS superconductors has a long history[1]. Early attempts to describe nonstationary superconductivity were based on the timedependent Ginzburg-Landau (TDGL) equation [2, 3, 4], which reduces the problem to the time evolution of a single collective order parameter $\Delta(t)$. The TDGL approach is valid only provided the system quickly reaches an equilibrium with the instantaneous value of $\Delta(t)$, i.e. a local equilibrium is established faster than the time scale of the order parameter variation, $\tau_{\Delta} \simeq 1 / \Delta$. This requirement limits the applicability of the TDGL to special situations where pair breaking dominates, e.g. due to a large concentration of magnetic impurities. An alternative to TDGL is the Boltzmann kinetic equation [5, 6] for the quasiparticle distribution function coupled to a self-consistent equation for $\Delta(t)$. This approach is justified only when external parameters change slowly on the $\tau_{\Delta}$ time scale, so that the system can be characterized by a quasiparticle distribution.

Is it possible to describe theoretically the dynamics of a BCS paired state in the nonadiabatic regime when external parameters change substantially on the $\tau_{\Delta}$ time scale? In particular, an important question is whether, following a sudden perturbation, the condensate reaches a steady state on a $\tau_{\Delta}$ time scale or on a much longer quasiparticle energy relaxation time scale $\tau_{\epsilon}$. In the nonadiabatic regime both TDGL and the Boltzmann kinetic equations fail and one has to deal with the coupled coherent dynamics of individual Cooper pairs. Recent studies 15, 16, 17, 18] of this outstanding problem were motivated by experiments on fermionic pairing in cold atomic alkali gases [7, 8]. The strength of pairing interactions in these systems can be fine tuned rapidly by a magnetic field, making it easier than in metals to access the nonadiabatic regime experimentally.

The main result of the present paper is an explicit general solution for the dynamics of the BCS model, which describes a spatially homogenous condensate at times $t \ll \tau_{\epsilon}$. We employ the usual BCS mean-field approximation, which is accurate when the number of Cooper pairs is large 10, 11]. It turns out that the mean-field BCS dynamics can be formulated as a nonlinear classical Hamiltonian problem. We obtain the exact solution for all initial conditions and a complete set of integrals of motion for the mean-field BCS dynamics.

In this paper we assume that the number of Cooper pairs in the system is arbitrary large, but finite. In this case the typical evolution at times $t \ll \tau_{\epsilon}$ is quasi-periodic with a large number of incommensurate frequencies. The condensate exhibits irregular multi-frequency oscillations ergodically exploring the part of the phase-space allowed by the conservation laws. The system returns arbitrarily close to its initial state at irregular time intervals. However, the return time diverges in the thermodynamic limit for most physical initial conditions, while the solution asymptotically reaches a steady state on the $\tau_{\Delta}$ time scale. The system thermalizes on a much larger energy relaxation time scale $\tau_{\epsilon}[9]$.

The dynamics of the BCS condensate following a sudden change of external parameters has been previously discussed by a number of authors 11, 12, 13, 14, 15, 16, 17, 18, 19. Most notably, a linear analysis around the BCS ground state has been performed [11, 12] and some simple particular solutions for the nonlinear mean-field dynamics in the context of superconductivity have been reported 14, 15]. We discuss below how these results fit into the general picture.

We begin our description of the nonequilibrium Cooper pairing in the non-dissipative regime, $t \ll \tau_{\epsilon}$, with the BCS model 20, 21, 22].

$$
\hat{H}_{B C S}=\sum_{j, \sigma} \epsilon_{j} \hat{c}_{j \sigma}^{\dagger} \hat{c}_{j \sigma}-g \sum_{j, q} \hat{c}_{j \uparrow}^{\dagger} \hat{c}_{j \downarrow}^{\dagger} \hat{c}_{q \downarrow} \hat{c}_{q \uparrow}
$$

where $\epsilon_{j}$ are single-particle energies. The pairing is between time reversed states $|j \uparrow\rangle$ and $|j \downarrow\rangle[23]$. Our goal is to determine the evolution of a state that was driven out of equilibrium at, say, $t=0$.

There are several equivalent ways to derive mean-field equations of motion. One can start with the BCS product 
state, $\prod_{j}\left(U_{j}(t)+V_{j}(t) c_{j \uparrow}^{\dagger} \hat{c}_{j \downarrow}^{\dagger}\right)|0\rangle$, and use Bogoliubovde Gennes equations for the time-dependent amplitudes $U_{j}(t)$ and $V_{j}(t)$. Alternatively, one can study the evolution of the normal, $G_{j}(t)=-i\left\langle\left[\hat{c}_{j \uparrow}(t), \hat{c}_{j \uparrow}^{\dagger}(t)\right]\right\rangle$, and anomalous, $F_{j}(t)=-i\left\langle\left[\hat{c}_{j \uparrow}(t), \hat{c}_{j \downarrow}(t)\right]\right\rangle$, Green's functions at coinciding times 12 .

The most convenient for us approach to the BCS meanfield dynamics is based on the Anderson pseudospin representation [11]. Within this approach the mean-field equations are Hamiltonian equations of motion for a classical spin chain. Pseudospin-1/2 operators are related to fermion creation and annihilation operators via $\hat{K}_{j}^{z}=\left(\hat{n}_{j \uparrow}+\hat{n}_{j \downarrow}-1\right) / 2$ and $\hat{K}_{j}^{-}=\hat{c}_{j \downarrow} \hat{c}_{j \uparrow}=\left(\hat{K}_{j}^{+}\right)^{\dagger}$. Pseudospins are defined on empty and doubly occupied (unblocked) single-particle orbitals $\epsilon_{j}$. Singly occupied orbitals are decoupled from the dynamics. For $n$ unblocked orbitals the Hamiltonian has the form

$$
\hat{H}_{B C S}=\sum_{j=0}^{n-1} 2 \epsilon_{j} \hat{K}_{j}^{z}-g \sum_{j, q} \hat{K}_{j}^{+} \hat{K}_{q}^{-}
$$

The mean field approximation is accurate 10, 11] in the thermodynamic limit due to the infinite range of interactions between spins in the Hamiltonian (2). Therefore, the effective field seen by each pseudospin in (2) can be replaced with its quantum mechanical average, $\mathbf{b}_{j}(t)=\left(-2 \Delta_{x}(t),-2 \Delta_{y}(t), 2 \epsilon_{j}\right)$, where $\Delta(t) \equiv \Delta_{x}(t)-$ $i \Delta_{y}(t) \equiv g \sum_{j}\left\langle\hat{K}_{j}^{-}(t)\right\rangle$ is the BCS gap function. In this approximation, each spin evolves in the self-consistent field: $\dot{\hat{\mathbf{K}}}_{j}=i\left[\hat{H}_{B C S}, \hat{\mathbf{K}}_{j}\right] \approx \mathbf{b}_{j} \times \hat{\mathbf{K}}_{j}$. Taking the quantum mechanical average of these equations with respect to the time-dependent state of the system, we obtain for $\mathbf{s}_{j}(t)=\left\langle\hat{\mathbf{K}}_{j}(t)\right\rangle$

$$
\dot{\mathbf{s}}_{j}=\mathbf{b}_{j} \times \mathbf{s}_{j} \quad \mathbf{b}_{j}=\left(-2 g J_{x},-2 g J_{y}, 2 \epsilon_{j}\right) \quad \mathbf{J}=\sum_{q=0}^{n-1} \mathbf{s}_{q}
$$

The components of the classical spins $s_{j}^{z}(t)$ and $s_{j}^{ \pm}=s_{j}^{x} \pm$ $i s_{j}^{y}$ are related to Bogoliubov amplitudes and equal times Green's functions as $2 s_{j}^{z}=\left|V_{j}\right|^{2}-\left|U_{j}\right|^{2}, s_{j}^{-}=\bar{U}_{j} V_{j}$ and $G_{j}(t)=i s_{j}^{z}(t), F_{j}(t)=i s_{j}^{-}(t)$, respectively. Evolution equations (3) conserve the square of the average for each spin: $d \mathbf{s}_{j}^{2} / d t=0$. If the spins initially were in a product state, $\mathbf{s}_{j}^{2}=1 / 4$. Note also that $\Delta(t)=g J_{-}(t)$.

One can check that Eqs. (3i) are equations of motion for a classical spin Hamiltonian

$$
H_{B C S}=\sum_{j=0}^{n-1} 2 \epsilon_{j} s_{j}^{z}-g \sum_{j, q} s_{j}^{+} s_{k}^{-}
$$

It means that Eqs. (3) are Hamilton equations $\dot{\mathbf{s}}_{j}=$ $\left\{H_{B C S}, \mathbf{s}_{j}\right\}$ derived from Hamiltonian (4) using the usual angular momentum Poisson brackets

$$
\left\{s_{j}^{a}, s_{k}^{b}\right\}=-\varepsilon_{a b c} \delta_{j k} s_{j}^{c}
$$

where $a, b$, and $c$ stand for spatial indexes $x, y$, and $z$. The classical model (4) can be obtained from its quantum counterpart (2) by replacing operators with classical dynamical variables and commutators with Poisson brackets.

Both the classical (4) and quantum models (1) and (2) are integrable 24, 25, 26. To show this, one can introduce a vector function (Lax-vector) of an auxiliary parameter $u$

$$
\mathbf{L}(u)=-\frac{\hat{\mathbf{z}}}{g}+\sum_{j} \frac{\mathbf{s}_{j}}{u-\epsilon_{j}},
$$

where $\hat{\mathbf{z}}$ is a unit vector along the $z$ axis. Poisson brackets between components of $\mathbf{L}(u)$ at different values of $u$ can be evaluated using Eq. (5).

$$
\left\{L^{a}(v), L^{b}(w)\right\}=\varepsilon_{a b c} \frac{L^{c}(v)-L^{c}(w)}{v-w}
$$

(Relations (7) hold for each term in (6) separately; all terms Poisson-commute with each other). It follows from Eq. (7) that the lengths of the Lax vector at different values of $u$ Poisson-commute:

$$
\left\{\mathbf{L}^{2}(v), \mathbf{L}^{2}(w)\right\}=0
$$

The scalar function $\mathbf{L}^{2}(u)$ can be represented in the form

$$
\mathbf{L}^{2}(u)=\frac{1}{g^{2}}+\sum_{j=0}^{n-1}\left(\frac{2 H_{j}}{u-\epsilon_{j}}+\frac{\mathbf{s}_{j}^{2}}{\left(u-\epsilon_{j}\right)^{2}}\right)
$$

where

$$
H_{j}=\sum_{k=0}^{n-1} \frac{\mathbf{s}_{j} \cdot \mathbf{s}_{k}}{\epsilon_{j}-\epsilon_{k}}-\frac{s_{j}^{z}}{g}
$$

Since Eq. (8) holds for any $v$ and $w$, all $H_{j}$ Poissoncommute with each other. Therefore, each $H_{j}$, as well as any algebraic combination of $H_{j}$, defines a classical model [27] that has $n$ degrees of freedom ( $n$ classical spins) and $n$ integrals of motion (including itself) and thus is integrable in the usual sense [28]. Note that the sum of $H_{j}$ is proportional to the $z$-component of the total spin $\mathbf{J}$, therefore $J_{z}$ is conserved by all $H_{j}$ and their combinations. Moreover, the following identity follows from Eqs. (410)

$$
H_{B C S}=-g \sum_{j} \epsilon_{j} H_{j}+\text { const }
$$

This implies that the classical BCS model (4) Poissoncommutes with all $H_{j}$ 's and thus is also integrable. Equations (10) and (11) can be straightforwardly quantized by replacing $\mathbf{s}_{j} \rightarrow \hat{\mathbf{K}}_{j}$. The resulting operators $\hat{H}_{j}$ all pairwise commute, thus showing the integrability of quantum models (1) and (2). 
To obtain the general solution for the mean-field dynamics of the BCS model (1), we follow the method of Ref. 29 and introduce $n-1$ separation variables $u_{k}$ as zeros of $L_{-}(u)=L_{x}(u)-i L_{y}(u)$, i.e. $\sum_{j} s_{j}^{-} /\left(u_{k}-\epsilon_{j}\right)=0$.

Equations of motion for the variables $u_{k}$ are 30

$$
\dot{u}_{k}=2 i \sqrt{Q_{2 n}\left(u_{k}\right)} \prod_{m \neq k}\left(u_{k}-u_{m}\right)^{-1}
$$

where $Q_{2 n}(u)$ is the spectral polynomial defined as

$$
Q_{2 n}(u)=g^{2} \mathbf{L}^{2}(u) \prod_{j}\left(u-\epsilon_{j}\right)^{2}
$$

By Eq. (9), the coefficients of $Q_{2 n}(u)$ depend only on the integrals of motion $H_{j}$.

Eqs. (12) constitute the well-known Jacobi's inversion problem solvable in terms of hyperelliptic theta functions [31]. Here we outline the final answer, the details will be reported elsewhere 30. Klenian $\sigma$ - and $\zeta$ functions of genus $G$ (in our case $G=n-1$ ) are defined as

$$
\begin{aligned}
& \zeta_{l}(\mathbf{x})=\frac{\partial \ln \sigma(\mathbf{x})}{\partial x_{l}} \quad \sigma(\mathbf{x})=\sum_{\mathbf{m} \in Z^{G}} \exp \left[S_{\mathbf{m}}(x) / 2\right] \\
& S_{\mathbf{m}}(x)=\mathbf{x} \cdot \eta \omega^{-1} \mathbf{x}+2 i \pi\left(\mathbf{m} \cdot \tau \mathbf{m}+\omega^{-1} \mathbf{x} \cdot \mathbf{m}\right)
\end{aligned}
$$

where the sum is over all $G$-dimensional integer vectors $\mathbf{m}, \tau=\omega^{\prime} \omega^{-1}$, and $\omega, \omega^{\prime}$, and $\eta$ are $G \times G$ matrices of periods (see below). The solution is

$$
\begin{gathered}
s_{j}^{-}(t)=\left\langle\hat{c}_{j \downarrow}(t) \hat{c}_{j \uparrow}(t)\right\rangle=J_{-}(t) r\left(\epsilon_{j}, t\right) \prod_{k \neq j} \frac{\epsilon_{j}}{\epsilon_{j}-\epsilon_{k}} \\
\Delta(t)=g J_{-}(t)=g \sum_{j} s_{j}^{-}(t)=c_{n} e^{-i \beta t} \frac{\sigma(\mathbf{x}+\mathbf{d})}{\sigma(\mathbf{x}-\mathbf{d})}
\end{gathered}
$$

Here $\mathbf{x}^{T}=i\left(c_{1}, \ldots, c_{n-2}, 2 t+c_{n-1}\right) ; \mathbf{d}$ is a vector of constants; $\beta=g J_{z}+\sum_{j} \epsilon_{j} ; c_{1}, \ldots, c_{n}$ are constants fixed by the initial conditions, and

$$
r(u, t)=1-\sum_{k=1}^{n-1}\left(\zeta_{k}(\mathbf{x}+\mathbf{d})-\zeta_{k}(\mathbf{x}-\mathbf{d})+a_{k}\right) u^{k-n}
$$

Constants $a_{k}$, the matrices of periods, constant vector $\mathbf{d}$, are all uniquely determined [32] by the spectral polynomial $Q_{2 n}(u)$, i.e. by integrals of motion.

The evolution of $\mathbf{s}_{j}(t)=\left\langle\hat{\mathbf{K}}_{j}(t)\right\rangle$ described by the general solution is typical of an integrable system 28. It is characterized by $n$ frequencies, which in our case can be determined [30] in terms of integrals of motion, and are typically incommensurate. Note that $|\Delta(t)|$ contains only $n-1$ frequencies. The typical dynamics is stable against perturbations destroying integrability 28.

Now let us discuss some particular solutions. There are two types of equilibrium states that play an important role in the dynamics. In normal states all spins are parallel to the $z$-axis, $2 \mathbf{s}_{j}^{z}= \pm 1$. Since $2 \mathbf{s}_{j}=\left\langle\hat{n}_{j}\right\rangle-1$, these states correspond to the ground state and excitations of the single-particle part of the Hamiltonian (1) (Fermi gas). They are stationary within the mean-field dynamics (3). For a finite system, they are non-stationary for the quantum Hamiltonian (11) and their short time dynamics is entirely driven by quantum corrections (cf. Refs. 33, 34).

The second type of equilibrium states are anomalous ones, which correspond to the BCS ground state and excitations. These states are obtained by aligning each spin in (4) self-consistently along the effective magnetic field acting on it. The self-consistency condition is the BCS gap equation. As $\mathbf{s}_{j}=\left\langle\hat{\mathbf{K}}_{j}\right\rangle$, one can obtain the BCS wave-function and energy spectrum from anomalous equilibrium configurations of classical spins $\mathbf{s}_{j}$.

It turns out that equilibrium states are a part of a more general scheme when the dynamics of $n$ spins degenerates to that of $m<n$ collective spins ( $m$-spin solutions) governed by the same Hamiltonian (4) only with $m$ spins and new parameters $\mu_{j}$ instead of $\epsilon_{j}$. Normal and anomalous states correspond to 0 - and 1-spin solutions, respectively. To construct $m$-spin solutions one has to take the Lax-vector (6) to be proportional to that of a system with $m$ spins $\mathbf{t}_{k}, \mathbf{L}(u)=\left[1+\sum_{j} b_{j} /\left(u-\epsilon_{j}\right)\right] \mathbf{L}_{\mathbf{t}}(u)$, where $b_{j}$ are time-independent constants, and $\mathbf{L}_{\mathbf{t}}(u)=$ $-\hat{\mathbf{z}} / g+\sum_{k} \mathbf{t}_{k} /\left(u-\mu_{k}\right)$. Then, $2(n-m)$ of $2 n$, typically distinct, roots of the spectral polynomial $Q_{2 n}(u)$ become doubly degenerate and $n-m$ separation variables $u_{k}$ are frozen in these double roots, which automatically solves the equations of motion for these $n-m$ variables. The dynamics is obtained by replacing $n \rightarrow m$ and $\epsilon_{k} \rightarrow \mu_{k}$ in Eqs. (14 17) and is characterized by $m<n$ typically incommensurate frequencies. For $m=2$ the solution is in terms of hyperelliptic functions of genus $G=m-1=1$, i.e. in terms of ordinary elliptic functions.

Now let us discuss the connection of our results with the previous work. The solutions for the mean field BCS dynamics obtained in Ref. 15 are 2-spin solutions in the above classification. They were used in Ref. 15 to describe the evolution beginning from a state infinitesimally close to the normal ground state. In our view, the dynamics in the vicinity of this state can have additional features and deserves further analysis.

The 2-spin solutions resemble the TDGL approach in that they describe the dynamics of all pairs in terms of only two collective degrees of freedom resulting in large amplitude single frequency (periodic) oscillations of the order parameter magnitude $|\Delta(t)|$. Mathematically, they lie on a $1 \mathrm{~d}$ curve of points in a multi-dimensional (infinitedimensional in the thermodynamic limit) space of possible values of integrals of motion. The situation with other few spin solutions is similar 35. In contrast, the general solution we obtained here typically has a large (infinite in the thermodynamic limit) number of incom- 
mensurate frequencies and a substantially reduced amplitude. The difference between the general and few spin solutions is clear in a linear analysis 11, 12] around the BCS ground state that displays normal modes with frequencies $\omega_{k}=2 \sqrt{\epsilon_{k}^{2}+\Delta_{0}^{2}}$, where $\epsilon_{k}$ are single-particle energies and $\Delta_{0}$ is the equilibrium order parameter. In the linear regime, the general solution becomes an arbitrary superposition of all normal modes, while few spin solutions single out all, but few modes. For example, 2spin solutions of Ref. 15 correspond to a single normal mode with a frequency $2 \Delta_{0}$.

In conclusion, we have obtained the explicit general solution for the mean-field dynamics of the BCS paired state and discussed a number of special cases including two types of equilibrium states and few spin solutions. A still open problem is to fully analyze the solution in the thermodynamic limit. It is also desirable to better understand the dynamics in the vicinity of normal states where quantum effects become important. Finally, it is interesting to identify experimental setups where peculiar features of the nonequilibrium Cooper pairing in the nonadiabatic regime can be observed such as e.g. cold Fermi gases.

We are grateful to I. Aleiner, V. Falko, L. Glazman, L. Levitov, A. Millis, A. Polyakov, and O. Tsyplyatyev for stimulating discussions. This research was supported by NSF DMR 0210575 and by ARO/ARDA (DAAD19-021-0039).

Note Added. Recently, we became aware of a publication 36] that is in agreement with some of our conclusions - that the initial dynamics of the normal ground state is driven by quantum corrections and that the system can reach a steady state at large times.

[1] N. B. Kopnin: Theory of Nonequilibrium Superconductivity, Clarendon Press, Oxford (2001).

[2] E. Abrahams and T. Tsuneto: Phys. Rev. 152, 416 (1966).

[3] A. Schmid: Phys. Kond. Mat. 5, 302 (1966).

[4] L. P. Gor'kov and G. M. Eliashberg: Sov. Phys. JETP 27, 328 (1968).

[5] O. Betbeder-Matibet and P. Nozieres: Ann. Phys. 51, 392 (1969).

[6] A. G. Aronov et. al.: Adv. Phys. 30, 539 (1981); In: Nonequilibrium Superconductivity (ed. D. N. Landenberg and A. I. Larkin), Elsevier (1986).

[7] M. W. Zwierlein et. al.: Phys. Rev. Lett. 92, 120403 (2004). C. A. Regal, M. Greiner, and D. S. Jin: Phys. Rev. Lett. 92, 040403 (2004).

[8] C. A. Regal, M. Greiner, and D. S. Jin: Phys. Rev. Lett. 92, 040403 (2004); M. W. Zwierlein et. al.: Phys. Rev. Lett. 92, 120403 (2004); J. Kinast et. al.: Phys. Rev. Lett. 92, 150402 (2004); M. W. Zwierlein et. al.: cond-mat/0412675

[9] E. A. Yuzbashyan, V. B. Kuznetsov, B. L. Altshuler: unpublished.
[10] R. W. Richardson: J. Math. Phys. 18, 1802 (1977).

[11] P. W. Anderson: Phys. Rev. 112, 1900 (1958).

[12] A. F. Volkov and Sh. M. Kogan: Sov. Phys. JETP 38, 1018 (1974).

[13] Yu. M. Gal'perin, V. I. Kozub, and B. Z. Spivak: Sov. Phys. JETP 54, 1126 (1981).

[14] V. S. Shumeiko: Doctoral Thesis, Institute for Low Temperature Physics and Engineering, Kharkov, 1990.

[15] R. A. Barankov, L. S. Levitov, and B. Z. Spivak: Phys. Rev. Lett. 93, 160401 (2004).

[16] A. V. Andreev, V. Gurarie, and L. Radzihovsky: Phys. Rev. Lett. 93, 130402 (2004).

[17] R. A. Barankov and L. S. Levitov: Phys. Rev. Lett. 93, 130403 (2004).

[18] M. H. Szymanska, B. D. Simons, and K. Burnett: Phys. Rev. Lett. 94 , 170402 (2005).

[19] M.H.S. Amin, E.V. Bezuglyi, A.S. Kijko, A.N. Omelyanchouk: Low Temp. Phys. 30, 661 (2004).

[20] J. Bardeen, L.N. Cooper, and J.R. Schriefer: Phys. Rev. 1081175 (1957).

[21] P. W. Anderson: J. Phys. Chem. Solids 11, 26 (1959).

[22] I. L. Kurland, I. L. Aleiner, and B. L. Altshuler: Phys. Rev. B 62, 14886 (2000).

[23] In the presence of spatial symmetries additional quantum numbers are needed to distinguish states. For example, if there is translational invariance, time-reversed pairs of states are $|j \uparrow\rangle=|\mathbf{p} \uparrow\rangle$ and $|j \downarrow\rangle=|-\mathbf{p} \downarrow\rangle$.

[24] R. W. Richardson and N. Sherman: Nucl. Phys. 52, 221 (1964); 52, 253 (1964).

[25] M. Gaudin: La fonction d'onde de Bethe, Masson, Paris (1983).

[26] M. C. Cambiaggio, A. M. F. Rivas, and M. Saraceno: Nucl. Phys. A 424, 157 (1997).

[27] Each of $H_{j}$ can be viewed as a Hamiltonian of a system of classical spins, these Hamiltonians are known as classical Gaudin magnets 25].

[28] V. I. Arnold: Mathematical Methods of Classical Mechanics, Springer-Verlag, New York, (1978); M. Tabor, Chaos and Integrability in Nonlinear Dynamics, Wiley, New York, (1989).

[29] E. K. Sklyanin: J. Sov. Math. 47, 2473 (1989); Progr. Theoret. Phys. Suppl. 118, 35 (1995); V. B. Kuznetsov: J. Math. Phys. 33, 3240, (1992).

[30] E. A. Yuzbashyan, B. L. Altshuler, V. B. Kuznetsov, V. Z. Enolskii: J. Phys. A 38, 7831, (2005); cond-mat/0407501

[31] E.D. Belokolos, A.I. Bobenko, V.Z. Enolskii, A.R. Its: Algebro-geometric approach to nonlinear-integrable equations, (Springer-Verlag, Berlin, 1994); D. Mumford: Tata Lectures on Theta, (Birkhauser, Boston, 1983, 1984) Vols. 1-2.

[32] J. C. Eilbeck, V. Z. Enolskii, and H. Holden: Proc. R. Soc. Lond. A 459, 1581, (2003).

[33] R. Bonifacio and G. Preparata: Phys. Rev. A 2, 336 (1970).

[34] A. Vardi, V. A. Yurovsky and J. R. Anglin: Phys. Rev. A 64, 063611 (2001).

[35] For certain initial conditions the dynamics in the thermodynamic limit can reduce to that of a few spin solution asymptotically at long times. For example, we expect $m=3,5, \ldots$ spin solutions at long times if the coupling is changed abruptly while the condensate is in an excited state.

[36] G.L. Warner and A. J. Leggett: Phys. Rev. B 71, 134514 
(2005). 